\title{
La storia naturale del Covid-19 nel setting delle cure primarie pediatriche in Italia. Uno studio osservazionale
}

\author{
GiacomoToffol*, Laura Reali**, Roberto Buzzetti*** \\ *Pediatra di famiglia, Pederobba (TV); **Pediatra di famiglia, Roma; ***Pediatra epidemiologo, Bergamo
}

\section{Introduzione}

La malattia Covid-19, che si è manifestata per la prima volta in Cina nel dicembre 2019, è comparsa ufficialmente in Italia a febbraio 2020 e da allora si è diffusamente estesa causando decine di migliaia di vittime, prevalentemente nelle classi di età più avanzata [1]. A partire da gennaio 2020 sono comparse in letteratura le prime segnalazioni di casi pediatrici e neonatologici inizialmente provenienti dalla Cina e poi anche dagli altri Paesi in cui il contagio si è diffuso [2-4]. I casi pediatrici registrati risultano compresi tra 1'1 e il 5\% dei casi totali $[5,6]$. Dai dati riportati in letteratura si evidenzia un basso numero di ricoveri e una mortalità bassissima [7-9].

In età pediatrica non vi sono ancora prove certe né sul rischio di contrarre la malattia né sul grado di contagiosità. Uno studio di coorte retrospettivo recentemente pubblicato, basato sui dati di 391 casi e 1.286 contatti stretti, sembrerebbe dimostrare che, almeno all'interno delle famiglie, i bambini hanno la stessa probabilità di essere infettati degli adulti, ma è stato pubblicato anche uno studio che smentisce questa ipotesi, e che afferma che il tasso di attacco secondario all'interno delle famiglie sia molto più basso nei bambini e ragazzi rispetto agli adulti (4\% contro $17 \%)$ [10]. Il grado di contagiosità dei bambini non è ancora ben noto [11]. Vi è inoltre una scarsa conoscenza della storia clinica dei casi di pazienti pediatrici non ricoverati e di quella dei contatti e non sono ancora ben conosciute infettività, durata dell'immunità acquisita, presenza/ rilevanza di sintomi neurologici (anosmia e ageusia, ma anche dolori muscolari, artralgie, ecc.), dermatologici (acrocianosi, eritema pernio-like, lesioni ulcerative delle estremità) e cardiocircolatori. Inoltre, anche se non è ancora nota la capacità dei bambini di contagiare altre persone, essi rappresentano sicuramente una fascia di età nella quale risulta praticamente impossibile il distanziamento fisico, con con- seguente esposizione a un elevato numero di contatti stretti [12]. Avere informazioni attendibili relativamente agli aspetti sopracitati sarebbe estremamente utile per comprendere appieno i rischi correlati a questa epidemia e per poter definire efficaci misure di controllo. Per ottenere le informazioni necessarie è importante effettuare degli studi anche in setting diversi da quelli ospedalieri $[13,14]$. Dato inoltre che al momento attuale non è stato ancora costituito nessuno score diagnostico validato che possa aiutare il clinico a sospettare questa malattia in una popolazione di età pediatrica, validarne uno sul campo potrebbe essere importante per migliorare le capacità diagnostica di questa malattia nel prossimo futuro.

\section{Obiettivi dello studio}

Si tratta di uno studio osservazionale, prospettico, multicentrico che intende valutare nel setting ambulatoriale delle cure primarie pediatriche l'incidenza e il decorso clinico di pazienti pediatrici affetti da $\mathrm{Co}^{-}$ vid-19 e dei loro contatti familiari ed extra-familiari.

Gli obiettivi secondari sono descrivere la durata dei sintomi; l'eventuale comparsa di sintomi nei contatti; l'esito degli eventuali esami diagnostici effettuati; il numero di nuovi casi che compaiono nei contatti, dopo la positività del caso (o contatto) arruolato; l'incidenza di sintomi rari associati di tipo dermatologico (acrocianosi, eritema pernio-like, lesioni ulcerative delle estremità), neurologico (ageusia e anosmia, artralgie, dolori muscolari), cardiocircolatorio e di altri eventuali sintomi nei pazienti positivi.

Con questo studio si intende inoltre valutare l'applicabilità sul campo di una scheda di sintomi per la diagnosi clinica di sospetto Covid-19. Non essendo presenti in letteratura schede validate per la diagnosi clinica di sospetto di Covid-19 in età pediatrica, per la costruzione di questa scheda sono state usate come partenza quella suggerita
dall'OMS nell'ultima revisione dei criteri per la definizione di caso sospetto di SARS [15] e come confronto la scheda per la diagnosi delle ILI utilizzata dalla rete italiana Sorveglianza Influenza (Influnet) [16] e la definizione di caso sospetto della circolare ministeriale n. 7.922 del 9 marzo 2020. I criteri clinici in esse contenuti sono stati poi integrati con tutti i segni e sintomi pubblicati in letteratura sulla infezione da SARS-CoV-2, dai più caratteristici ai più rari descritti (Tabella 1 ).

\section{Materiali e metodi}

Lo studio prevede l'arruolamento dei bambini e ragazzi con età inferiore a 14 anni di ambo i sessi, afferenti presso gli ambulatori dei PLS da ottobre 2020 a marzo 2021, che rientrino in almeno una delle seguenti condizioni:

- tampone positivo per SARS-CoV-2;

- caratteristiche cliniche di caso ed esecuzione di tampone (indipendentemente dall'esito);

- contatti stretti di Covid-19.

Per tutti i soggetti arruolati verranno rilevate le seguenti variabili: età, sesso, peso, altezza, condizioni cliniche generali, patologie croniche, tosse, febbre, dispnea, vomito, diarrea, lesioni dermatologiche, sintomi neurologici, muscolari, cardiocircolatori. I bambini verranno visitati e/o valutati in ambulatorio o a casa o in teleconsulto in relazione alla gravità del caso, secondo la normale pratica professionale del pediatra delle cure primarie. Verrà richiesta l'effettuazione di un tampone per la diagnosi di Covid-19 secondo le regole definite dalla succitata circolare ministeriale presso i centri localmente dedicati dalle rispettive Regioni (USCAR, SISP, ecc.).

Dopo l'arruolamento avremo quindi tre gruppi di bambini e ragazzi: un gruppo di "casi" confermati dal tampone; un gruppo di "casi" non confermati dal tampone; un gruppo di contatti stretti, conviventi con genitori o parenti positivi. I genitori 
TABELLA 1. Criteri per la definizione di caso sospetto di Covid-19.

Presenza di almeno uno dei seguenti segni e sintomi (in ottemperanza alla circolare ministeriali n. 7.922 del 9 marzo 2020) in assenza di elementi di eziologia e clinica che siano chiaramente indicativi di origine diversa da Covid-19:

\begin{tabular}{|l|l|l}
\hline Febbre (T.C. $\left.>0=a 37,5^{\circ} \mathrm{C}\right)$ & Tosse & Dispnea
\end{tabular}

Eventualmente associati, come riportato dalla letteratura selezionata a uno o più dei seguenti:

\begin{tabular}{|l|l|l|}
\hline Tachipnea/dispnea & Dolore toracico & Rinite \\
\hline Faringodinia & Congiuntivite & Dolore oculare \\
\hline Diarrea & Vomito & Dolore addominale \\
\hline Astenia & Mialgia & Artralgia \\
\hline Cefalea & Ageusia & Anosmia \\
\hline Acrocianosi & Eritema pernio-like & $\begin{array}{l}\text { Lesioni ulcerative delle estre- } \\
\text { mità }\end{array}$ \\
\hline Rash & Cheilite & \\
\hline
\end{tabular}

di questi bambini e ragazzi risponderanno a un questionario anonimo erogato online attraverso Google moduli, appositamente costruito, per fornire le informazioni necessarie al follow-up, che avrà una durata variabile tra 14 e 28 giorni a seconda dei casi. Su tutti i dati raccolti verrà poi condotta un'analisi descrittiva relativamente a: numero totale dei partecipanti, loro caratteristiche (demografiche, cliniche, sociali), informazioni sulle esposizioni, durata dei sintomi, eventuale comparsa di sintomi di positività nei contatti, incidenza dei nuovi casi che compaiono nei contatti.

\section{Numerosità del campione e risultati attesi}

A oggi i casi pediatrici confermati in Italia sono 20.133 [17]. Dai dati presenti in letteratura si evince che per ogni caso diagnosticato ci possano essere tra i 6 e i 20 casi non diagnosticati $[18,19]$. Ponendo arbitrariamente questo valore a 10 possiamo stimare che a oggi si siano verificati in Italia circa 200.000 casi confermati di infezione da SARS-CoV-2 nei bambini (su circa 7.400.000 bambini fino a 14 anni, stima approssimativa dai dati ISTAT). Reclutando un campione di 74.000 bambini (corrispondente a circa un centinaio di pediatri), ci si aspetta un centinaio di nuovi casi di Covid-19/mese durante i sei mesi dello studio, 600 in totale nei sei mesi dello studio. L'incidenza stimata di 100 casi/mese avrebbe dei limiti di confidenza al 95\% di circa 80-120 casi/mese; corrispondenti a 1,35 casi/mese ogni 1.000 bambini (IC95\% 1,08-1,62).

Con questa numerosità campionaria sarà possibile stimare con buona precisione alcune caratteristiche rilevate tra i soggetti: una caratteristica che si presentasse con una frequenza del 10, 30, 50 per cento avrebbe come limiti dell'IC95\% rispettivamente $\mathrm{di}+/-2,4 \%, 3,7 \%, 4 \%$.

Per quanto riguarda l'obiettivo principale - stimare la incidenza e valutare l'epidemiologia della patologia Covid-19 - tale incidenza verrà stimata nel campione saggiandone anche l'associazione con le principali covariate previste nello studio, sia con test sulle medie o sulle mediane (a seconda delle distribuzioni) che con i test sulle proporzioni, quali il test chi quadrato e il test esatto di Fisher. La significatività statistica verrà saggiata con alfa $=0,05$. Verrà inoltre valutata (confrontando la frequenza delle diverse associazioni di segni e sintomi nei casi positivi e negativi al tampone) la capacità diagnostica della scheda clinica precostituita, per individuare clinicamente i casi sospetti.

\section{Considerazioni finali e commenti}

A nostra conoscenza questo è il primo studio osservazionale sulla storia naturale del Covid-19 nel setting delle cure primarie italiane. Dato che a quanto risulta dalla letteratura la maggior parte dei bambini e ragazzi affetti da questa patologia non necessitano di cure ospedaliere, si tratta di un ambito molto importante per raccogliere informazioni sulla reale incidenza di questa patologia in età pediatrica. Conoscere meglio le dinamiche di questa patologia, e in modo particolare la contagiosità dei bambini e ragazzi, potrà fornire ai clinici e ai decisori politici informazioni importanti per garantire i diritti dei minori, compresa la possibilità di frequentare la scuola.

Siamo consapevoli del fatto che non sarà uno studio semplice da condurre, sia per una certa complessità intrinseca del disegno, che prevede una decisa e continua collaborazione dei pediatri partecipanti e delle famiglie, sia soprattutto per le difficoltà che stiamo vivendo nel momento in cui scriviamo (speriamo non ulteriormente aggravate al momento dell'uscita di questo articolo), legate alla diffusione del SARS-CoV-2, che mette in seria crisi medici e famiglie oltre che l'organizzazione sanitaria e l'intera società. Anche la diversità di tracciamento dei casi sospetti che si osserva attualmente nelle diverse Regioni italiane e che si basa su protocolli locali a volte inutilmente farraginosi potrà essere un ulteriore fattore di complessità. Riteniamo tuttavia utile condurre lo studio certi di ottenere comunque dei risultati che possano condurre a una migliore gestione di questa patologia in età pediatrica.

$凶$ gitoffol@gmail.com

La bibliografia è consultabile online. 
1. Livingston E, Bucher K.Coronavirus disease 2019 (COVID-19) in Italy. JAMA 2000:323;1335-1335.

2. Dong Y, Mo X, Hu Y et al. Epidemiological characteristics of 2143 pediatric patients with 2019 coronavirus disease in China. Pediatrics 2020.

3. CDC COVID-19 Response Team. Coronavirus disease 2019 in children - United States, February 12 April 2 , 2020. MMWR Morb Mortal Wkly Rep 2020;69:422-6.

4. Castagnoli R, Votto M, Licari A. Severe Acute Respiratory Syndrome Coronavirus 2 (SARS-CoV-2) Infection in Children and Adolescents. A Systematic Review. JAMA Pediatrics2020.

5. Ludvigsson JF. Systematic review of COVID-19 in children show milder cases and a better prognosis than adults. Acta Paediatrica2020;109:1088-95.

6. Kammoun R, Masmoudi K. Paediatricaspects of COVID-19. An update. RespirMed Res 2020;78:100765.

7. Castagnoli R, Votto M, Licari A et al. Severe Acute RespiratorySyndrome Coronavirus 2 (SARSCoV-2)

Infection in children and adolescents. A systematicreview. JAMA Pediatr 2020.

8. Lu X, Zhang L, Du H, et al. SARSCoV-2 infection in Children. N Engl J Med 2020;382:1663-5.

9. Liguoro I, Pilotto C, Bonanni M et al. SARS-COV-2 infection in children and newborns: a systematic review. Eur J Pediatrics2020;179:1029-46.

10. Liu Y, Gu Z, Xia S et al. \&quot; What are the underlying transmission patterns of covid-19 outbreak?-an agespecific social contact characterization.\&quot; EClinicalMedicine (2020): 100354.

11. Bi Q, Wu Y, Mei S, et al. Epidemiology and transmission of COVID-19 in 391 cases and 1286 of their close contacts in Shenzhen, China: a retrospective cohort study. The Lancet InfectiousDiseases. 2020.

12. Zhang J, Litvinova M, Liang Y et al.Changes in contact patterns shape the dynamics of the COVID-19 outbreak in China. Science.ZHANG, Juanjuan, et al. Changes in contact patterns shape the dynamics of the COVID-19 outbreak in China. Science, 2020.

13. Lipsitch M, Swerdlo DL, Finelli L. Defining the epidemiology of Covid-19—-studies needed. New England journal of medicine, 2000;382:1194-6.

14. NIH begins study to quantify undetected cases of coronavirus infection. https://www.nih.gov/newsevents/news-releases/nih-begins-study-quantify-undetected-cases-coronavirus-infection

15. WHO guidelines for the global surveillance of severe acute respiratory syndrome (SARS) Updated recommendations October 2004

https://www.who.int/csr/resources/publications/WHO_CDS_CSR_ARO_2004_1/en/

16. Istituto Superiore di Sanità Sistema di Sorveglianza Integrata dell’Influenza https://old.iss.it/site/RMI/influnet/pagine/Documenti.aspxISS Epidemia COVID-19 Aggiornamento nazionale 6 ottobre 2020 - ore 11:00. Data pubblicazione: 9 ottobre 2020

17. ISS Epidemia COVID-19. Aggiornamento nazionale 6 ottobre 2020

18. Lachmann, A. Correcting under-reported COVID-19 case numbers. 2020.medRxiv.

19. Fanelli D. Piazza F. Analysis and forecast of COVID-19 spreading in China, Italy and France. Chaos,

Solitons\&amp;Fractals, 2020;134:109761. 